\title{
23. CENOZOIC RADIOLARIANS FROM DEEP SEA DRILLING PROJECT LEG 40
}

\author{
Nicklas G. Pisias and T.C. Moore, Jr., Graduate School of Oceanography, \\ University of Rhode Island, Kingston, Rhode Island
}

\section{BIOSTRATIGRAPHIC ZONATION}

Only at Sites 360 and 362 are upper Miocene to Pleistocene radiolarians sufficiently abundant for detailed biostratigraphy. Because of the general absence of radiolarians at Leg 40 sites, it is difficult to define an adequate zonation for the southeastern Atlantic. However, the stratigraphic distribution of some radiolarian species at Site 362 seems to be similar to that in the North Pacific, and we therefore use the upper Miocene to Pliocene biostratigraphy of Kling (1973).

Miocene: The upper Miocene is represented by the Stichocorys peregrina Zone. The base of this zone (not identified at Site 362) is marked by the first occurrence of Stichocorys peregrina, and its top by the last occurrence of this species.

Pliocene: The base of the Pliocene Lamprocyrtis heteroporos Zone coincides with the top of the $S$. peregrina Zone. As discussed by Kling (1973), the Miocene-Pliocene boundary occurs within the $S$. peregrina Zone. In the Pacific Coast region, the boundary is tentatively taken as the lowest occurrence of Lamprocyrtis heteroporos. Thus, in the North Pacific, the ranges of $S$. peregrina and $L$. heteroporos overlap. The same is observed at Site 362. In the Antarctic, however, the base of the Pliocene is marked by the first appearance of Cycladophora davisiana (Chen, 1975). Based on calcareous nannoplankton and foraminifers, the Miocene-Pliocene boundary lies between Cores 362-13 and 15. At Site 362, as in the North Pacific, the first occurrences of $C$. davisiana and $L$. heteroporos are not synchronous. $C$. davisiana first appears in Section 362-14-4 and $L$. heteroporos in Section 362-18-4. Thus, the first appearance of $C$. davisiana more closely approximates the MiocenePliocene boundary, and at Site $362 \mathrm{~L}$. heteroporos ranges down into the Miocene.

Pleistocene: The species used to identify the Pleistocene in other parts of the world were not found at Site 362 and thus the Pliocene/Pleistocene boundary cannot be identified.

\section{SITE SUMMARIES}

\section{Site 360}

Radiolarians are in general very scarce and poorly preserved. Species present in Cores 1 to 10 (Stichocory:s delmontensis, Ommatartus penultimus, and Ommatartus antepenultimus) suggest that these sediments are upper Miocene.

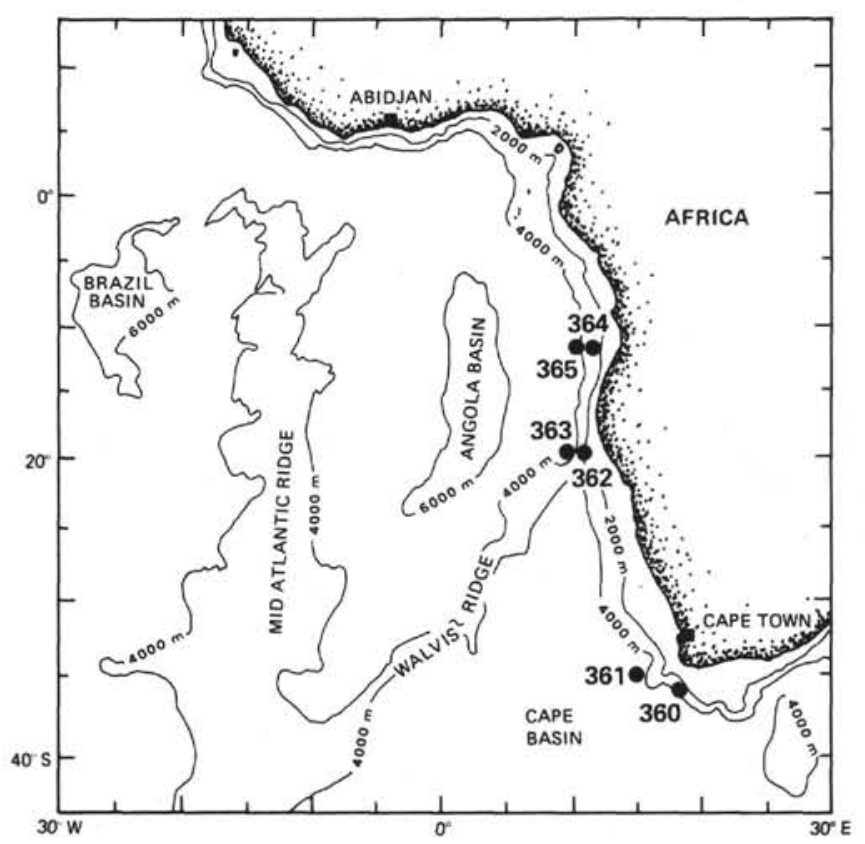

Figure 1. Location of DSDP Leg 40 Sites 360 to 365.

\section{Site 361}

No Cenozoic radiolarians were found at this site.

\section{Site $\mathbf{3 6 2}$}

Radiolarians were found in Cores 1 to 24 from Site 362 , indicating that the sediments range from upper Miocene to possibly Pleistocene. Species tabulations and estimates of radiolarian abundances and preservation are shown in Table 1.

In general, the abundance of radiolarians is low through the upper Miocene and lower Pliocene, with preservation poor to moderately good. Samples from Cores 6 through 2 show increasing abundance of radiolarians with a marked increase in Cycladophora davisiana. The preservation in these samples is better than in samples above and below. The topmost sample examined, $362-1-4,99-101 \mathrm{~cm}$, has almost no radiolarians.

As previously discussed, the Miocene-Pliocene boundary is placed within Core 14 of Site 362 . The identification of the Pliocene-Pleistocene boundary by means of radiolarians is not possible at this site, but the presence of Axoprunum angelinum in Sample 362-2-4, $99 \mathrm{~cm}$, gives it a minimum age of 400,000 years. 
TABLE 1

Radiolarians at Site 362

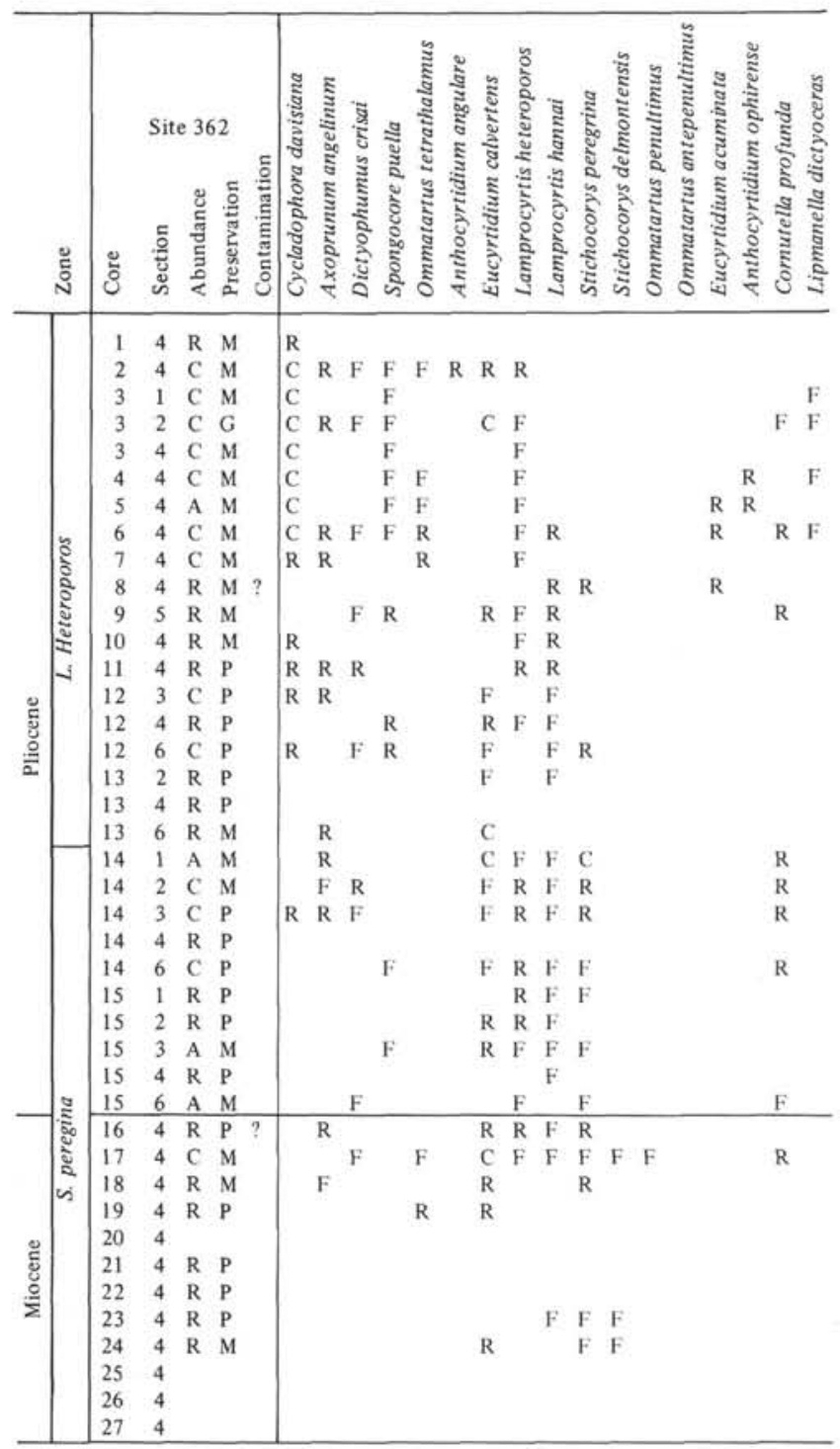

Note: $\mathrm{A}=$ abundant, $\mathrm{C}=$ common, $\mathrm{F}=\mathrm{few}, \mathrm{R}=$ rare, $\mathrm{G}=$ good preservation, $\mathrm{M}=$ moderate, $\mathrm{P}=$ poor.

\section{Site 364}

Radiolarians were found only in the core-catcher sample of Core 6. Specimens in this sample are sparse and highly fragmented. Identifiable specimens of Artophormis gracillis, Lithocyclia crux, and an unnamed forerunner of this latter species (as in Plate 5, Figure 1), suggest that this sample is similar in age to Section 365-7-1 and belongs to the Theocyrtis tuberosa Zone.

\section{Site 365}

Radiolarians were found only in Sample 7-1, 32-35 $\mathrm{cm}$ from Site 365 . This sample contains several radiolarian and diatom species that have been replaced by pyrite. Radiolarians present include:

Artophormis gracilis Riedel (Plate 4, Figure 3)

Lithocyclia angustum (Riedel) (Plate 4, Figure 4)

Lithocyclia crux Moore

Dorcadospyris spp.
(Plate 5, Figures 2, 3)

(Plate 4, Figures 1, 2)
On this basis, the sample is assigned to the Theocyrtis tuberosa Zone (Riedel and Sanfilippo, 1970a; Moore, 1971). Preservation is poor, and specimens of Dorcadospyris cannot be easily identified to the species level. Surprisingly, this assemblage does not appear to include $T$. tuberosa, a relatively abundant species in the tropical Pacific; however, the base of $T$. tuberosa Zone is defined by the first appearance of $L$. angustum, and the range of $L$. crux is contained within the upper part of this zone. The presence of these species together with others listed above and figured in Plates 4 and 5 allow the assignment of the sample to the middle to upper part of this basal Oligocene zone.

\section{SYSTEMATICS}

Order POLYCYSTINA Ehrenberg

POLYCYSTINA Ehrenberg, 1838, emend. Riedel, 1967b, p. 291

Suborder SPUMELLARIA Ehrenberg, 1875

Family ACTINOMMIDAE Haeckel, 1862, emend. Riedel 1967b

\section{Genus AXOPRUNUM Haeckel}

Axoprunum Haeckel, 1887, p. 298. Type species (by monotype) Axoprunum stauraxonium Haeckel (1887, p. 298, pl. 48, fig. 4)

\section{Axoprunum angelinum (Campbell and Clark) (Plate 1, Figure 4)}

Stylosphaera angelina Campbell and Clark, 1944, p. 12, pl. 1, fig. 14-20.

Stylatractus sp. Hays, 1965. p. 167, pl. 1, fig. 6.

Stylatractus universus Hayes, 1970, p. 215, pl. 1, fig. 1, 2: Kling, 1971, p. 1086, pl. I, fig. 7.

Axoprunum angelinum Kling, 1973, p. 634, pl. 12, fig. 13-16, pl. 6, fig. 14-18.

Genus OMMATARTUS Haeckel 1881

Ommatartus Haeckel, I881, p. 463, Type species indicated by Riedel and Funnel, 1964, p. 311.

\section{Ommatartus antepenultimus Riedel and Sanfilippo} (Plate 1, Figure 3)

Panarium antepenultimus conditional manuscript name proposed by Riedel and Funnell, 1964, p. 311.

Ommatartus antepenultimus Riedel and Sanfilippo, 1970, pl. 14, fig. 4 .

Ommatartus penultimus (Riedel) (Plate 1, Figure 2)

Panarium penultimus Riedel, 1957, p. 76, pl. 1, fig. 1.

Ommatartus penultimus (Riedel) Riedel and Sanfilippo (senu. stricto), 1970, p. 521.

Ommatartus tetrathalamus (Haeckel) (Plate 1, Figure 1)

Panartus tetrathalamus Haeckel, 1887, p. 378, pl. 40, fig. 3, Nigrini, 1967, p. 30-32, pl. 2, fig. 4a-4d.

Ommatartus tetrathalamus (Haeckel), Renz, 1973, p. 158, pl. 1, fig. 6.

\section{Family COCCODISCADAE Haeckel, 1862}

\section{Genus LITHOCYCLIA Ehrenberg}

Lithocyclia Ehrenberg 1847 chart to p. 385. Type species (by monotype) Lithocyclia ocellus Ehrenberg (1854, p. 136, fig. 30; 1873 , p. $240 ; 1875$, pl. 29 , fig. 3 ) 
Lithocyclia crux Moore

(Plate 5, Figures 2, 3)

Lithocyclia crux Moore 1971, p. 737, pl. 6, fig. 4.

\section{Lithocyclia angustum (Riedel) \\ (Plate 4, Figure 4)}

Trigonactura angusta Riedel, 1959, p. 292, pl. I, fig. 6

Lithocyclia angustum (Riedel) Riedel and Sanfilippo, 1970, pl. 13, fig. $1,2$.

Family SPONGODISCIDAE Haeckel, 1862, emend. Riedel, 1967b

\section{Genus SPONGOCORE Haeckel}

Sponcore velata Haeckel (1887, p. 346)

\section{Spongocore puella Haeckel (Plate 1, Figure 5)}

Spongocore puella Haeckel, 1887, p. 347, pl. 48, fig. 6; Benson, 1964 , pl. 1, fig. 21; Nigrini, 1970, p. 168, pl. 2, fig. 3.

Spongocore lata Campbell and Clark, 1944, p. 22, pl, 3, fig. 5, 6

Spongocore puer Campbell and Clark, 1944, p. 22, pl. 3, fig. 7-9.

Suborder NASSALARIA Ehrenberg, 1875

Family ACANTHODESMIIDAE, Haeckel, 1862

Acanthodemiidae Haeckel: Riedel 1967b, p. 296.

Genus DORCADOSPYRIS Haeckel, 1862

(Plate 4, Figures 1, 2)

Dorcadospyris Haeckel, 1881, p. 141. Type species (indicated by Campbell 1954, p. 112

Dorcadospyris dentata Haeckel (1887, p. 1040, pl. 85, fig. 6).

Dorcadospyris Haeckel; emend. Goll, 1969, p. 335.

Family THEORPERIDA Haeckel, 1881, emend. Riedel, 1967b

\section{Genus ARTOPHORMIS Haecke}

Arthophormis Haeckel 1881, p. 438. Type species (indicated by Campbell, 1954, p. 139), Artophormis horrida Haeckel (1887, p. 1458 , pl. 74 , fig. 2 )

\section{Artophormis gracilis Riedel}

(Plate 4, Figure 3)

Artophormis gracilis Riedel, 1959, p. 300, pl. 2, fig. 12, 13.

\section{Genus CORNUTELLA Ehrenberg}

Cormutella Ehrenberg, 1838, p. 138. Type species (indicated by Campbell, 1954), Cornutella clathrata Ehrenberg (1838, p. 129 : 1844, pl. 77; 1856, pl. 22, fig. 39).

\section{Cornutella profunda Ehrenberg}

(Plate 1, Figure 5)

Cornutella profunda Ehrenberg, 1858, p. 31; Riedel, 1958, p. 232, pl. 3, fig. 1, 2; Nigrini, 1967, p. 60 , pl. 6, fig. 5a-5c.

Cornutella palverdensis Campbell and Clark, 1944, p. 40, pl. 5 , fig. $7,20,23,24,25$.

Cornutella clathrata $\beta$ profunda Ehrenberg, 1956, pl. 35B, fig. 21: Bailey, 1856, p. 2, pl. 1, fig. 23.

\section{Genus DICTYOPHIMUS Ehrenberg}

Dictyophimus Ehrenberg, 1847, p. 54. Type species (by monotype), Dictyophimus crisiae. genus.

Nigrini (1967, p. 68) discusses the correct type species for this

\section{Dictyophimus crisiae Ehrenberg}

(Plate 2, Figure 5)

Dictyophimus crisiae Ehrenberg, 1854, p. 241, Nigrini, 1967, p. 66 , pl. 6 , fig. $7 \mathrm{a}, 7 \mathrm{~b}$.
Pterocorys hirundo Haeckel, Casey, 1971, pl. 23.1, fig. 6, 7.; Petrushevskaya, 1967 (Partim), p. 115, fig. 4, 5).

Pterocorys hirundo Haeckel, Riedel, 1958, p. 238, pl. 3, fig. 1I, pl. 4, fig. 1; Petrushevskaya, 1967 (Partim), p. 115, fig. 1-3.

\section{Genus EUCYRTIDIUM Ehrenberg, 1847}

\section{Eucyrtidium calvertense Martin}

(Plate 1, Figure 7)

Eucyrtidium calvertense Martin, 1904, p. 450, pl. 130, fig. 5; Hays, 1965 , p. 181, pl. 3, fig. 4; 1970, p. 213, pl. I, fig. 6.

\section{Eucyrtidium acuminatum (Ehrenberg)}

Lithocampe acuminatum Ehrenberg, 1844, p. 84.

Eucyrtidium acuminatum (Ehrenberg), 1847, p. 43: Nigrini, 1967. p. 81 , pl. 8 , fig. 3 a, 3 b.

Genus LIPMANELLA Loeblich and Tappan, 1961

Lipmanella Loeblich and Tappan, 1961, p. 229 (=Dictyocera. Haeckel, 1862). Species Lithornithium dictyoceres Haeckel (= Dictyoceras virchowii $\mathrm{Haeckel}$ ).

\section{Lipmanella dictyoceras (Haeckel)}

$$
\text { (Plate 3, Figure 4) }
$$

Lithornithium dictyoceras Haeckel, 1860, p. 840.

Dictyoceras virchowii $\mathrm{Haeckel,} 1862$, p. 333, pl. 8, fig. 1-5.

Lipmanella virchowii (Haeckel) Petrushevskaya, 1971, p. 220. fig. 198.

Lipmanella dictyoceras (Haeckel) Kling, (1973), p. 636.

Genus STICHOCORYS Haeckel, 1881

\section{Stichocorys delmontensis (Campbell and Clark)}

Eucyrtidium delmontensis Campbell and Clark, 1944, p. 56, pl. 7. fig. 19,20 .

Stichocorys delmontensis (Campbell and Clark), Sanfilippo and Riedel, 1970, p. 451, pl. 1, fig. 9.

\section{Stichocorys peregrina (Riedel)}

(Plate 3, Figures 1-3)

Eucyrtidium elongatum peregrinum Riedel, 1953, p. 812, p. 1.85 , fig. 2.

Stichocorys peregrina (Riedel) Sanfilippo and Riedel, 1970, p. 451, pl. I, fig. 10.

Remarks: As was observed in the north Pacific (Kling, 1973), the specimens of $S$. peregrina found at Sites 360 and 362 have a less markedly conical third segment than in equatorial regions, with many not conical at all. The same criterion used by Kling (1973) to separate $S$. peregrina and $S$. delmontensis is used here, namely, specimens with the fourth segment as wide as the third, and thicker walled than the fifth and subsequent segments distinguish $S$. peregrina.

\section{Genus CYCLADOPHORA Ehrenberg, 1847}

\section{Cycladophora davisiana Ehrenberg}

$$
\text { (Plate 4, Figure 5) }
$$

Cycladophora davisiana Ehrenberg, 1862, p. 297; 1873, pl. 2, fig. 11 Petrushevskaya, 1967, p. 122, fig. 69, 70.

Theocalyptra davisiana (Ehrenberg) Riedel, 1958, p. 239, pl. 4, fig. 2 , 3 , text-fig. 10.

Family PTEROCORYIDAE Haeckel, 1881, emend. Riedel, 1967b

\section{Genus LAMPROCYRTIS Kling}

Lamprocyrtis hannai (Campbell and Clark)

(Plate 2, Figure 4)

Calocyclas hannai Campbell and Clark, 1944, p. 69, fig. 21, 22; Casey et al., 1972, pl. 3, fig. 10-22.

Calocyclas margatensis Campbell and Clark, 1944, p. 47, pl. 6 , fig. 17, 18; Casey et al., 1972, pl. 3, fig. 10-22. 
Lamprocyrtis hannai Kling, 1973, p. 638, pl. 5, fig. 12-14, pl. 12, fig. 10-14.

\section{Lamprocyrtis heteroporos (Hays)}

(Plate 2, Figures 1, 2)

Lamprocyclas heteroporos Hays, 1965, p. 179, pl. 3, fig. 1; Hays, 1970 , p. 214 , pl. 1, fig. 3 ; Kling, 1971, p. 1088 , pl. 1, fig. 1 .

Lamprocyrtis heteroporos (Hays) Kling, 1973, p. 639, pl. 5, fig. 1921 , pl. 15 , fig. 6 .

\section{Genus ANTHOCYRTIDIUM Haeckel, 1881}

\section{Anthocyrtidium angulare Nigrini}

Anthocyrtidium angulare Nigrini, 1971, p. 445, pl. 34.1, fig. 3a, 3b. Anthocyrtidium ophirense (Ehrenberg).

Anthocyrtidium cineraria Haeckel, 1887, p. 1278, pl. 62, fig. 16.

Anthocyrtidium ophirense (Ehrenberg) Nigrini, 1967, p. 56, pl. 6, fig. 3 .

\section{REFERENCES}

Bailey, J.W., 1856. Notice of microscopic forms found in the soundings of the Sea of Kamtchatka: Mar. J. Sci., v. 22, p. 1.

Benson, R.N., 1964. Preliminary report on Radiolaria in Recent sediments of the Gulf of California in marine geology of the Gulf of California-A Symposium: Am. Assoc. Petrol. Geol. Mem. 3, p. 398.

Campbell, A.S., 1954. Radiolaria. In Moore, R.C. (Ed.), Treatise on invertebrate paleontology: Kansas (Univ. Kansas Press and Geol. Soc. Am). Pt. D., Protista 3.11.

Campbell, A.S. and Clark, B.L., 1944. Miocene radiolarian faunas from southern California; Geol. Soc. Am., Spec. Paper 51 .

Casey, R.E., 1971. Radiolarians as indicators of past and present water masses, In Funnell, B.M. and Riedel, W.R. (Eds.), The micropaleontology of oceans: Cambridge (Cambridge Univ. Press), p. 331.

Casey, R.E., Price, A.B., and Swift, C.A., 1972. Radiolarian definition and paleoecology of the late Miocene to early Pliocene in southern California biostratigraphic symposium, March 9-10, 1972, Bakersfield, California: In Stiemeyer, E.H. (Ed.), The Pacific Coast Miocene, Pacific Sec.: Soc. Econ. Paleontol. Mineral., p. 226.

Chen, P.-H., 1975. Antarctic Radiolaria. In Hayes, D.E., Frakes, L.A., et al., Initial Reports of the Deep Sea Drilling Project, Volume 28: Washington (U.S. Government Printing Office), p. 437.

Ehrenberg, C.G., 1862. Die Tiefgrund-Verhaltnisse des ozeans am Eingange der Davisstrasse und bei island: Monatsber: Kgl. Preuss. Akad. Wiss. Berlin, p. 275. 1838. Über die Bildung der Kriedefelsen und des Kriedemergels durch unsichtbare Organismen: $\mathrm{Kgl}$. Akad. Wiss. Berlin, Abh. Jahre 1838.

1844. Über 2 neue Lager von Gebirgsmassen aus Infusorien als Meeres-Absatz in Nord-Amerika und eine Vergleichung derselben mit den organischen KriedeGebilden in Europe und Afrika: Kgl. Preuss. Akad. Wiss. Berlin, Ber. Jahre 1844

1847. Über die mikroskopischen Kieselschaligen Polycystinen als machtige Gebirgsmasse von Barbados: Kgl. Preuss. Akad. Wiss. Berlin, Ber. Jahre 1847.

374. 1854-1856. Mikrogeologie: Leipzip (Voss), p. 1-

1872. Mikrogeologischen Studien als Zusammenfassung der Beobachtungen des kleinsten Lebens der Meeres Tiefgrunde aller Zonen und dessen geologischen Einfluss: Kgl. Preuss. Akad. Wiss. Berlin, Monatsber, Jahre 1872
1873. Grossere Felsproben des PolycystinenMergels von Barbados mit weiteren Erlauterungen: Ber. Kgl. Preuss. Akad. Wiss. Berlin. Jahre 1873. 1875. Fortsetzung der mikrogeologischen Studien als Gesammt-Uebersicht der Mikroskopischen Palaontologie gleichartig analysirter Gebirgsarten der Erde, mit Specieller Rucksicht auf den PolycystinenMergel von Barbados: Abh. Kgl. Wiss. Berlin. Jahre 1875, p. 1 .

Goll, R.M., 1969. Classification and phylogeny of Cenozoic Trissocyclidae (Radiolaria) in the Pacific and Caribbean Basins. Part. II: J. Paleontol. v. 43, p. 322.

Haeckel, E., 1860. Abbildungen und Diagnosen neuer Gattungen und Arten von lebenden Radilarien des Mittelmeeres: Kgl. Preuss Akad. Wiss., Montasber. Jahre 1860 .

1862. Die Radiolarien, Eine Monographie.

1881. Entwurf eines Radiolarien-Systmes auf Grund von Studien der Challenger-Radiolarien: Jena. Z. Med. Naturwiss. v. 15 (new ser. 8), p. 418.

1887. Report on the Radiolaria collected by H.M.S. Challenger during the years 1873-76: Rept. Voy. Challenger, Zool., v. 18.

Hays, J.D., 1965. Radiolaria and late Tertiary and Quaternary history of Antarctic seas: Am. Geophys. Union Antarc. Res. Ser. 5, p. 125.

1970. Stratigraphy and evolutionary trends of Radiolaria in North Pacific deep sea sediments. In Hays, J.D. (Ed.) Geological investigations of the North Pacific: Geol. Soc. Am. Mem. 126.

Kling, S.A., 1971. Radiolaria. In Fischer, A.G., Heezen, B.C., et al., Initial Reports of the Deep Sea Drilling Project, Volume 6: Washington (U.S. Government Printing Office), p. 1060.

1973. Radiolaria from the Eastern North Pacific, Deep Sea Drilling Project Leg 18. In Kulm, L.D., von Heune, R., et al., Initial Reports of the Deep Sea Drilling Project, Volume 18. Washington (U.S. Government Printing Office), p. 617.

Loeblich, A.R., Jr., and Tappan, Helen, 1961. Remarks on the systematics of the Sarkodina (Protozoa), renamed homonyms and new and validated genera: Biol. Soc. Washington Proc., v. 74, p. 213.

Moore, T.C., Jr., 1971. Radiolaria, Leg 8, Deep Sea Drilling Project. In Tracey, J.I., Jr., Sutton, G.H. et al., Initial Reports of the Deep Sea Drilling Project, Volume 8: Washington (U.S. Government Printing Office), p. 727.

Nigrini, C., 1967. Radiolaria in pelagic sediments from the Indian and Atlantic oceans: Scripps Inst. Oceanogr. Bull., Univ. Calif., v. 11.

1970. Radiolarian assemblages in the North Pacific and their application to a study of Quaternary sediments in Core V29-130. In Hays, J.D. (Ed.), Geological investigations of the North Pacific: Geol. Soc. Am., Mem. 126 , p. 139

1971. Radiolarian zones in the Quaternary of the Equatorial Pacific Ocean. In Funnel, B.M. and Riedel, W.R. (Eds.), The micropaleontology of oceans: Cambridge (Cambridge University Press), p. 443.

Petrushevskaya, M.G., 1967. Radiolyarii otrvadov Spummelaria i Nassellaria antarkticheskoi oblasti (Antarctic spummeline and nasseline radiolarians). Issled. Faunv Morei 4 (12): Rez. biol. issled. Sov. Antarkt. Eksped. 1955-58, v. 3, p. 5.

1971. Radiolyarii Nassellaria $v$ planktone microvogo okeana. (Radiolaria Nassellaria of the world ocean). Issled. Fauny Morei IX (XVII): Akad. Nauk., S.S.S.R., Izdat. "Nauka". 
Renz, G.W., 1973. The distribution and ecology of Radiolaria in the Central Pacific, plankton and surface sediments: Ph.D. Thesis, University of California.

Riedel, W.R., 1957. Radiolaria: preliminary stratigraphy. Rept. Swedish Deep-Sea Exped. v. 6, p. 59.

1958. Radiolaria in Antarctic sediments. Rept. B.A.N.Z. Antarct. Res. Exped., Ser. B, v. 6, p. 217.

1959. Oligocene and lower Miocene Radiolaria in tropical Pacific sediments: Micropaleontology, v. 5, p. 285 . 1967a. Some new families of Radiolaria: Geol. Soc. London Proc., p. 148.

1967b. Subclass Radiolaria. In Harland, et al. (Eds.), The fossil record: London (Geol. Soc. London), p. 291.

Riedel, W.R. and Sanfilippo, A., 1970. Radiolaria, Leg 4, Deep Sea Drilling Project. In Bader, R.G., Gerard, R.D., et al., Initial Reports of the Deep Sea Drilling Project, Volume 4: Washington (U.S. Government Printing Office), p. 503.

Sanfilippo, A. and Riedel, W.R., 1970. Post-Eocene "closed" theoperid radiolarians: Micropaleontology, v. 16, p. 446. 
PLATE 1

$($ Scale $=100 \mu \mathrm{m})$

Figure $1 \quad$ Ommatartus tetrathalamus.

Sample 360-4-3, 99-101 cm.

Figure $2 \quad$ Ommatartus penultimus.

Sample 362-4-4, 99-101 cm.

Figure $3 \quad$ Ommatartus antepenultimus (broken).

Sample 360-10-2, 99-101 cm.

Figure 4 Axopunum angelinum.

Sample 362-7-4, 99-101 cm.

Figure $5 \quad$ Cornutella profunda.

Sample 362-6-4, 99-101 cm.

Figure $6 \quad$ Spongocore puella.

Sample 362-14-4, 99-101 cm.

Figure $7 \quad$ Eucyrtidium calvertense.

Sample 362-24-4, 99-101 cm.

PLATE 2

$($ Scale $=100 \mu \mathrm{m})$

Figure 1 Lamprocyrtis heteroporos.

Sample 362-5-4, 99-101 cm.

Figure $2 \quad$ L. heteroporos.

Sample $362-4-4,99-101 \mathrm{~cm}$.

Figure 3 L. heteroporos.

Sample 362-15-3, 99-101 cm.

Figure 4 Lamprocyrtis hannai.

Sample 362-24-4, 99-101 cm.

Figure 5 Dictyophimus crisiae.

Sample 362-6-4, 99-101 cm.

(see p. 852) 
PLATE 1
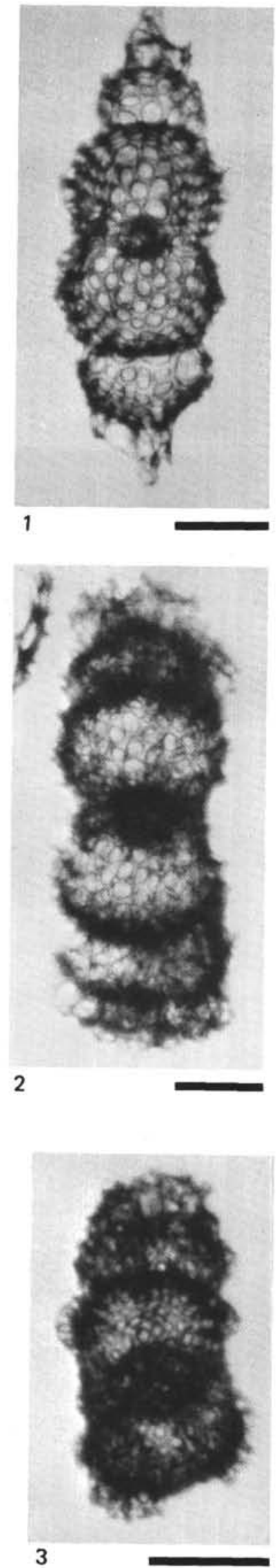
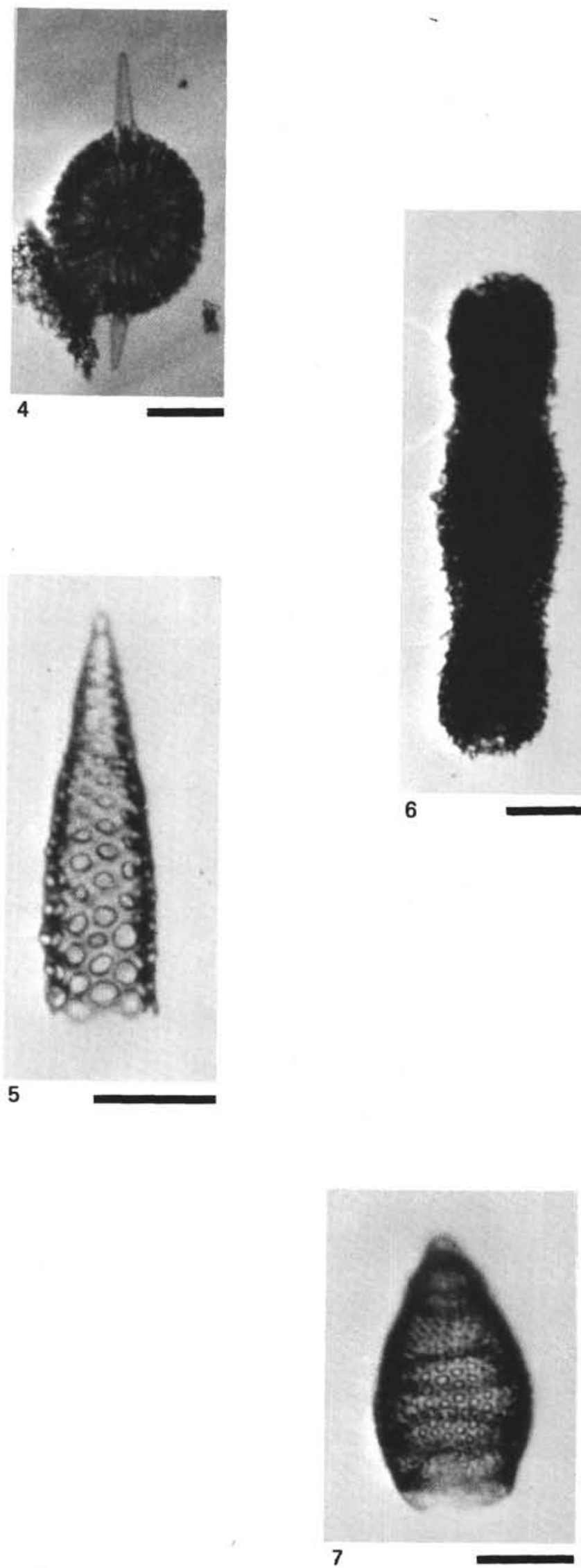
PLATE 2
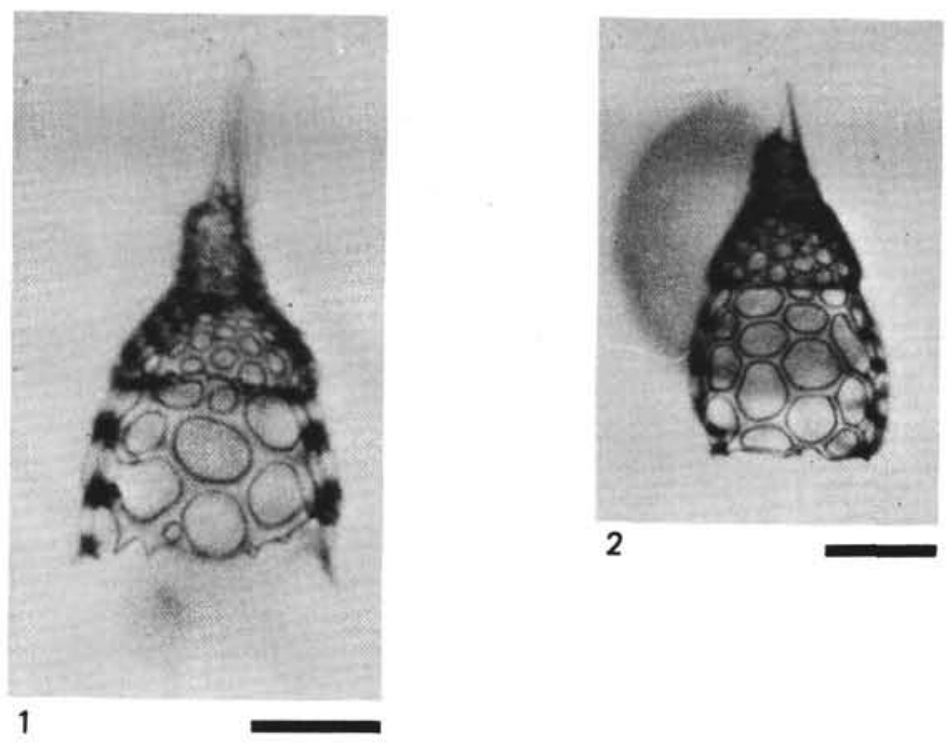
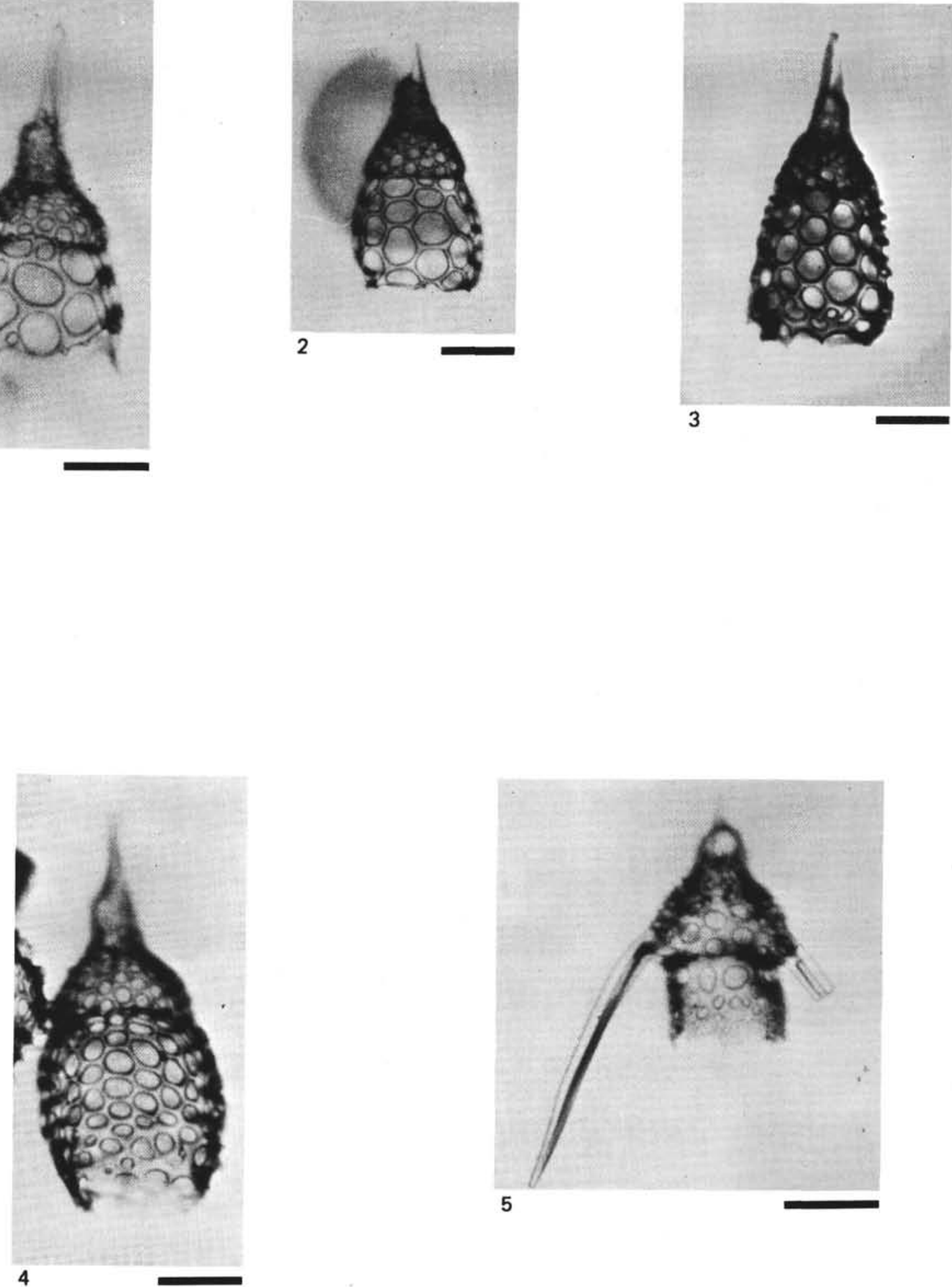
PLATE 3

$($ Scales $=100 \mu \mathrm{m})$

Figure 1

Stichocorys peregrina.

Sample 362-24-4, 99-101 cm.

Figure 2 Stichocorys peregrina.

Sample 362-24-4, 99-101 cm.

Figure 3 Stichocorys peregrina.

Sample 362-18-4, 99-101 cm.

Figure 4

Lipmanella dictyoceras.

Sample 362-6-4, 99-101 cm.

Figure 5 Cycladophora davisiana.

Sample 362-4-4, 99-101 cm.

Figure 6 Dictyophimus crisiae.

Sample 362-2-4, 99-101 cm.

(see p. 854)

PLATE 4

(Sample 365-7-1, 32-35 cm)

Figure 1 Dorcadospyris sp. $(648 \times)$.

Figure 2 Dorcadospyris sp. $(658 \times)$.

Figure $3 \quad$ Artophormis gracilis $(624 \times)$.

Figure $4 \quad$ Lithocyclia angustum $(428 \times)$.

(see p. 855)

PLATE 5

(Sample 365-7-1, 32-35 cm)

Figure $1 \quad$ Lithocyclia c.f. La crux $(484 \times)$.

Figure 2 Lithocyclia crux (396×).

Figure 3 Lithocyclia crux (420X).

(see p. 856) 
N. G. PISIAS, T. C. MOORE, JR.
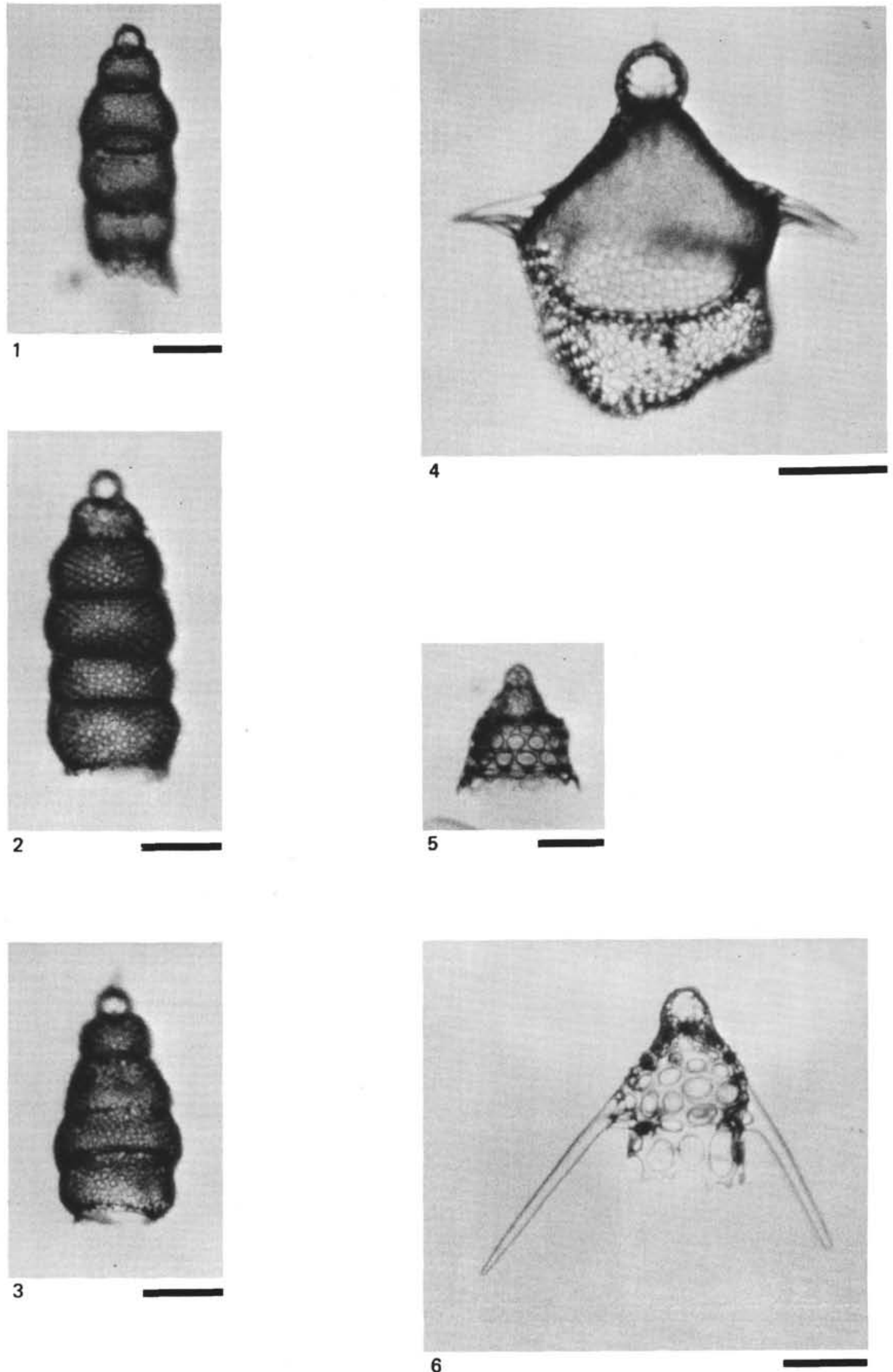
PLATE 4

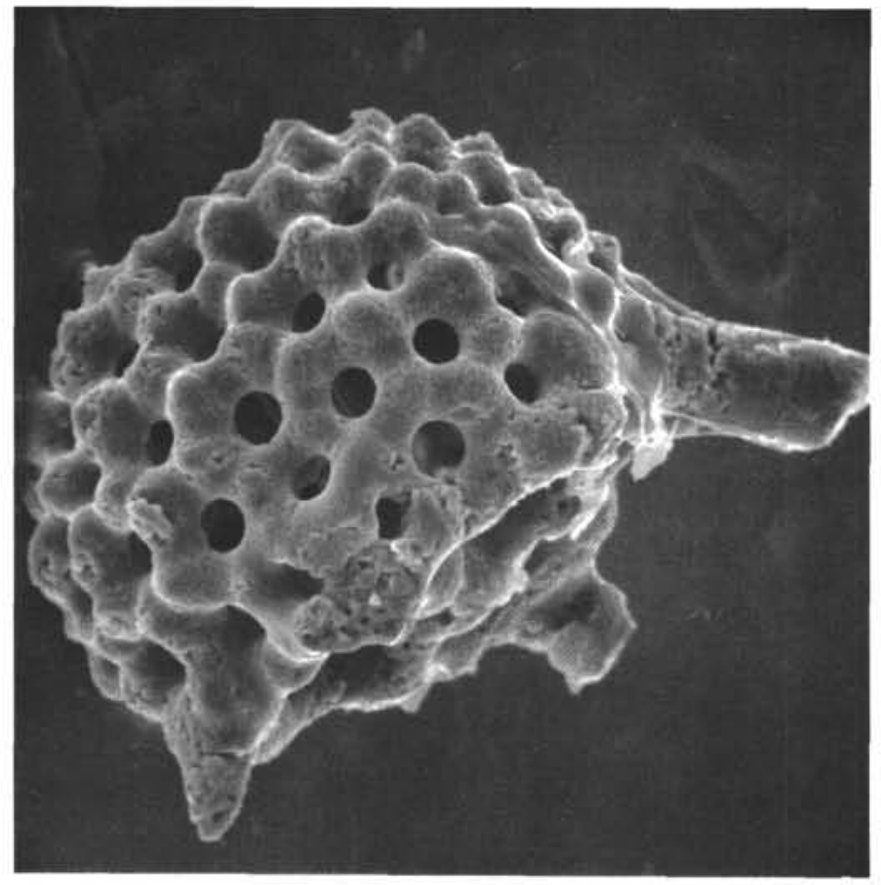

1

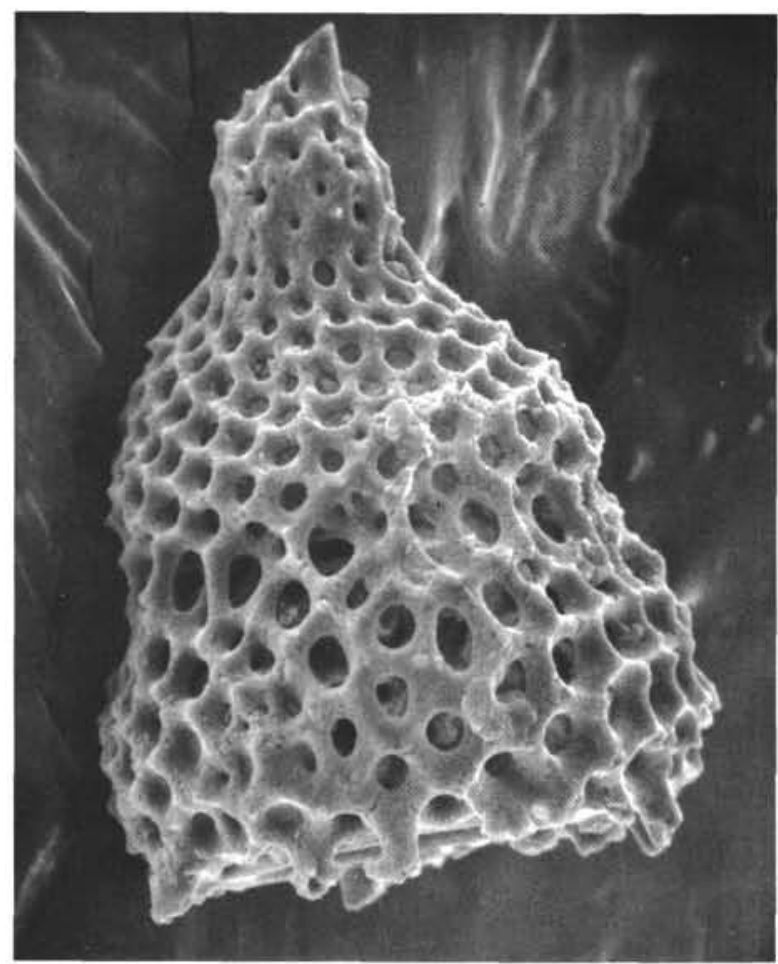

3

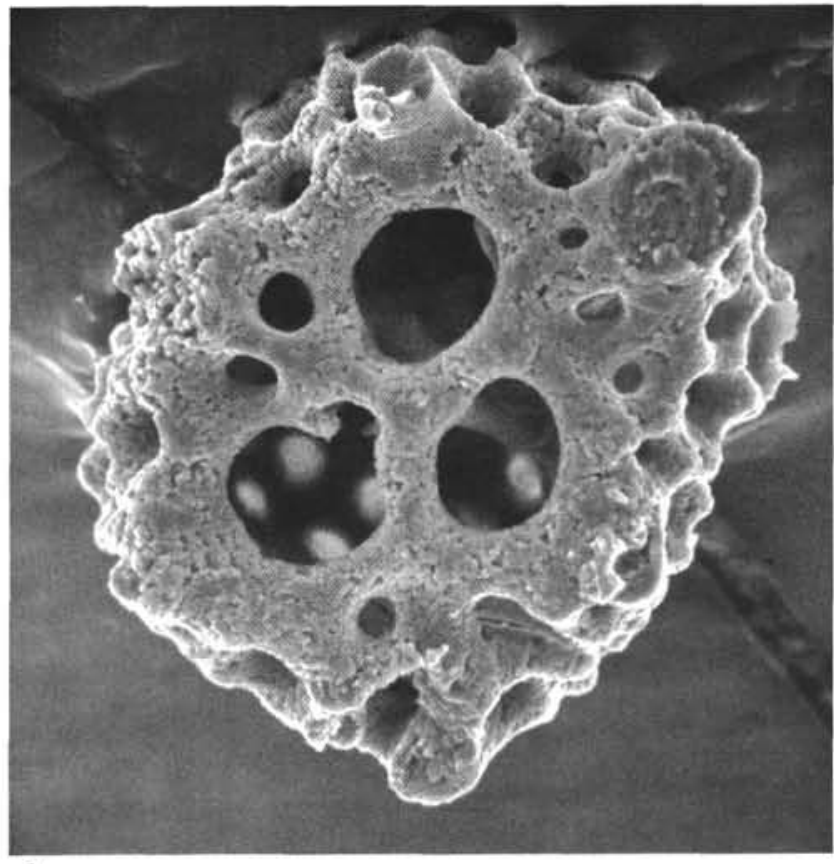

2

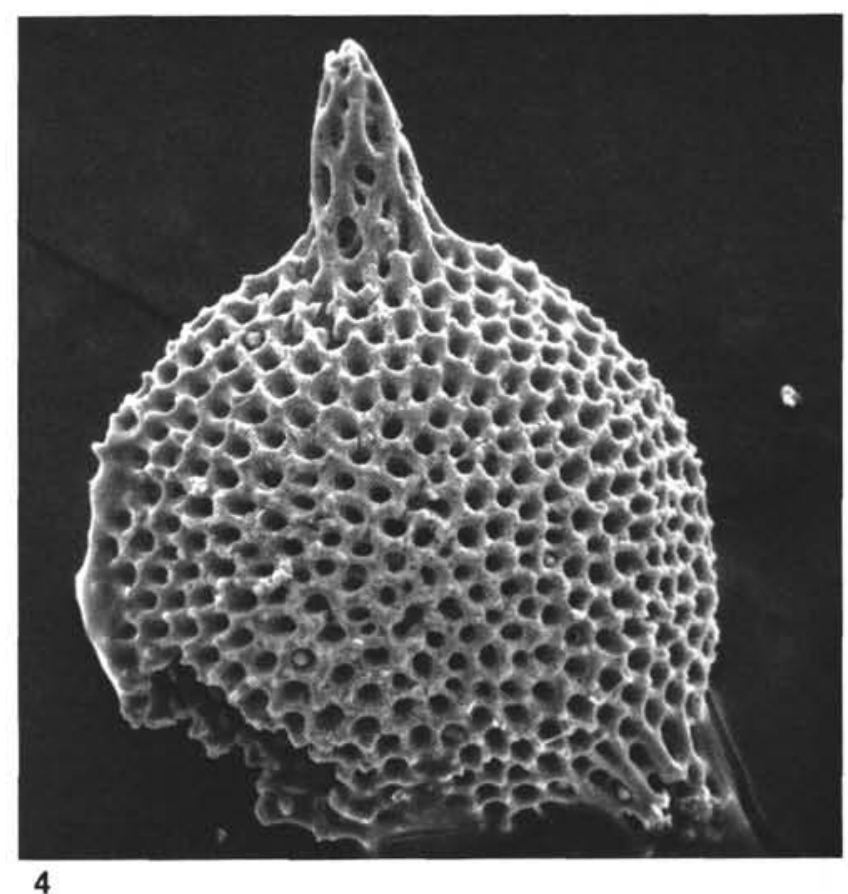

4 
N. G. PISIAS, T. C. MOORE, JR.

PLATE 5
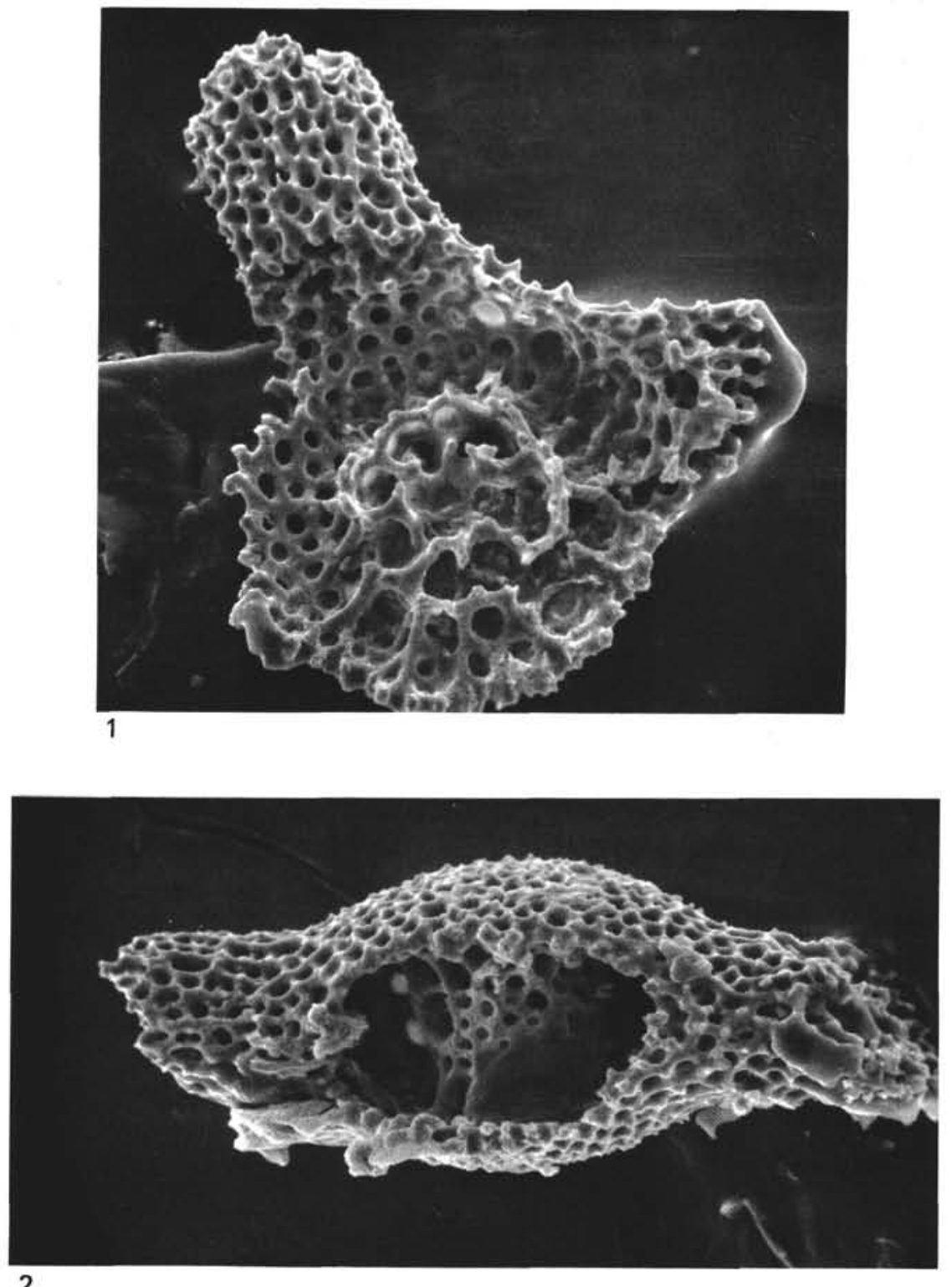

2

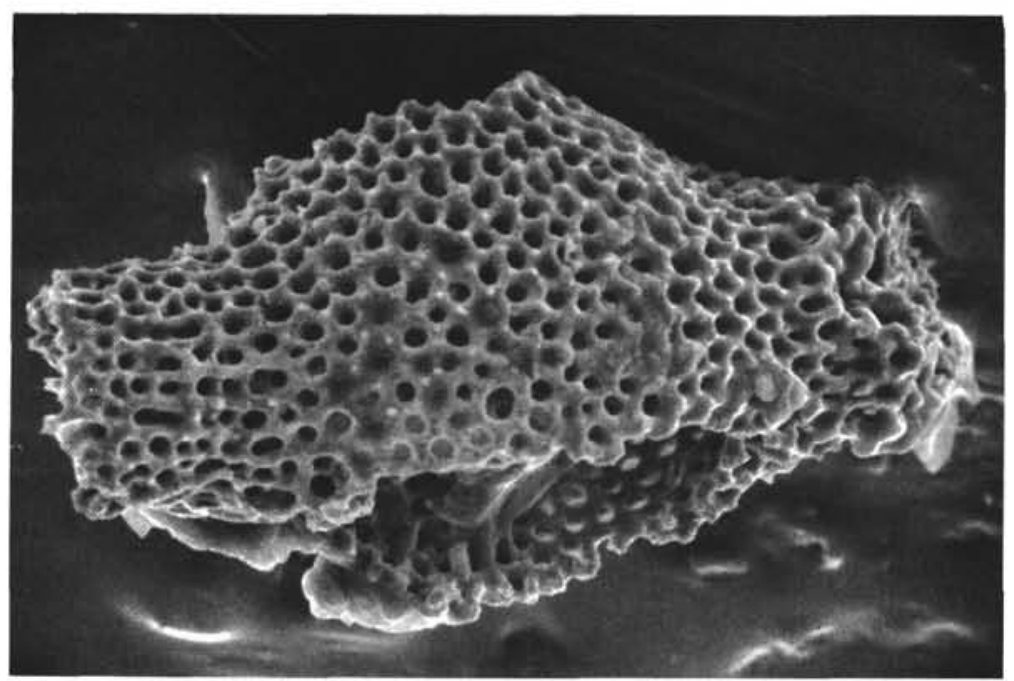

